\title{
Future perspectives in heart pathology diagnosis and therapy. how we can use the micro RNA?
}

\begin{abstract}
Heart disease is the number one cause of death worldwide with the number of people diagnosed ever increasing due to an ageing population also has a great social and economic impact. Recently, the investigation of new method of diagnosis and treatment of cardiovascular diseases are become topical, but also the elucidation of they own mechanisms are in focus. Beside of known signaling pathways which involved in heart pathology the miRNA is actively investigated as potential regulator of molecular mechanisms in heart including disease. Based on short review of experimental data we proposed that miRNA has a huge potency for early heart pathology diagnosis as markers.
\end{abstract}

Keywords: heart, microRNAs, signaling networks, heart pathology, early diagnosis
Volume 2 Issue 5 - 2017

\section{Oksana O Piven}

Department of Humans Genetic, Institute of Molecular Biology and Genetics of NAS of Ukraine, Ukraine

\author{
Correspondence: Oksana O Piven, Department of Humans \\ Genetic, Institute of Molecular Biology and Genetics of NAS of \\ Ukraine, 03680, Kyiv, I 50 Akad. Zabolotnogostr, Ukraine, Tel 380 \\ 44 526-I I-69, Fax + 38044 526-07-59, \\ Email o.o.piven@imbg.org.ua
}

Received: May 17, 2017| Published: July 25, 2017

\section{Introduction}

Heart disease is the number one cause of death worldwide with the number of people diagnosed ever increasing due to an ageing population and increased rates of obesity and diabetes, posing a significant health care burden. Heart failure is a major concern in modern society. According to the report by the European Heart Network and European Society of Cardiology in 2016, more than three-fifths of all CVD deaths occur in those over the age of 75 years, 1.4 million people under the age of 75 and just under 700000 under the age of 65 die from CVD in Europe each year. More men (0.9 million) than women ( 0.5 million) die from CVD before the age of $75 .{ }^{1}$ These make up to $47 \%$ of the cause of deaths in Europe and $40 \%$ in the EU.

It is important to note that diseases of the cardiovascular system not only threaten the health of European population but also has a great social and economic impact, because they often lead to significant degeneration of the quality of life and impairment of individual capabilities. ${ }^{1-3}$

This has cost the EU economy close to $€ 196$ billion per year, most of which goes to health care costs. Thus, such dramatic expansion of heart disease cases motivates us for looking new approaches of heart pathology diagnosis and therapy. ${ }^{3}$

The adult heart is a dynamic organ capable of significant remodeling and hypertrophic growth as a means of adapting function to altered workloads or injury. Hemodynamic stress or neuroendocrine signaling associated with myocardial infarction, hypertension, aorticstenosis, and valvular dysfunction evoke a pathologic remodeling response through the activation of intracellular signaling pathways and transcriptional mediators in cardiac myocytes. ${ }^{2,4}$ Activation of these molecular pathways may initially augment cardiac output (adaptive hypertrophy), however, prolonged hypertrophy (pathological or maladaptive hypertrophy) leads to heart failure, arrhythmia and sudden cardiac death.

Several lines of studies have shown that in response to pathologic stress there is partial reactivation of genes that promote embryonic and foetal heart development. Thus, a thorough understanding of the mechanism that controls cardiogenesis and adult heart maturation is essential not only for developmental biology, but also for understanding the molecular mechanism underlying the pathophysiology of heart disease. For the present time we have a line of experimental data for signaling networks impact in heart function maintains and heart disease development. Among of them MAPK, PI3K/AKT/mTOR, G-protein coupled receptor, canonical Wnt and cAMP/PKA signaling pathways. $^{4-7}$

All of them in focus of scientist such as they regulate heart size, metabolism, contractive function and cardiomyocytes growth. Definitely, the present progress in the identification of genes and signaling pathways involved in mediating of heart remodelling is impressive. However due to the overall complexity of hypertrophic remodelling and myocardial repair, further characterization of the underlying molecular mechanisms of mentioned process is needed. Widely known that heart failure as well as other heart disease are not a single loss-of-function genetic abnormality, its raiser more complex process. Moreover the fine-tuning gene expression may represent another feasible avenue via microRNAs (miRs).

MicroRNAs (miRNAs) are short, single-stranded, non-coding, highly conserved posttranscriptional regulators found in a variety of tissues including circulating blood. MicroRNAs regulate gene expression post transcriptionally through inhibiting translation form and/or inducing degradation of specific RNAs. ${ }^{8}$ Recently it was shown that MicroRNAs contribute to cardiac development and remodelling, and found in cardiac tissue show dynamic changes in the setting of heart disease, suggesting their involvement in the regulation of cardiovascular disease (CVD). Experimental studies with mouse and human samples have emphasized the importance of individual miRNAs in different forms of CVD, including myocardial infarction (mirR-1, miR-133a, miR-208a, and miR-499), atrial and ventricular arrhythmias (miR-1, miR-133, and miR-328), fibrosis (miR-21 and miR-29), and ventricular hypertrophy (miR-208 and miR-133). ${ }^{9-12}$

Thus, association between circulating miRNAs and CVD risk factors (hypertension and diabetes mellitus) was shown, and there is an emerging literature associating specific miRNAs with coronary artery disease, myocardial infarction, and heart failure. ${ }^{13-15}$ As known, intronic miRNA are coded within a host gene, and very often has ability to regulate the pathways similar to those of the protein encoded 
by that gene. ${ }^{16}$ That's why intracellular levels of miRNAs may provide information about regulatory pathways involved in mediating the initiation of heart remodelling. And some experimental data are in the line with this idea: it's known that MiR133a can regulate the ionchannel-encoding genes HCN2 and HCN4, ${ }^{11}$ mir-208a is encoded in the intron of the a-myosin heavy chain gene and indirectly regulates b-myosin heavy chain and connexin-40 expression; ${ }^{17}$ and miR-499 is intronic to the myosin gene Myh7b. ${ }^{17}$ Recently was shown that miRNA can modulate the canonical signaling activity in heart: miR-19a/b can specifically repress canonical Wnt through FZD4 and LRP6; ${ }^{18}$ miR26a leads to cardiomyocytes hypertrophy trough target GSK3b. ${ }^{19}$

Summarizing these massive of experimental data we can assume that miRNAs are able to regulate principally important signaling pathways as well as genes which up-regulated during heart pathology. Really, this idea is supported by single case researches which indicate miRNAs involving in heart disease. Thus, analysis of circulating miRNAs in blood samples from 29 patients and 10 healthy asymptomatic persons showed that high level of miR-499 in plasma may be a significant biomarker of myocardial infarction in humans. ${ }^{20}$ Analysis of 25 patients with ST elevation myocardial infarction (STEMI) revealed that miR-208b strongly correlated with troponin level and the ejection fraction. ${ }^{21}$ In the same study authors registered high level of miR-1, miR-133a, miR-208b and miR-499-5p in plasma of patients, and what more interesting, miR-1and miR-133a was detected in urine of patient with STEMI. ${ }^{21}$ This data indicate that not only circulating miRNAs in blood but also in other fluids like urine are very promising for heart pathology diagnosis. Other group of authors identified four miRNAs which strongly discriminated patients with chronic heart failure from controls: miR-660-3p, miR-665, miR$1285-3 \mathrm{p}$ and miR-4491.22

\section{Conclusion}

Summarizing this short review we should note that miRNA have reshaped our view of how cardiac gene expression is regulated, but deeper understanding of interlink between miRNA and keys signaling in heart remodelling has a great benefit not only for basic research but for clinical using also. In fact, using of circulatory miRNAs is very promising for effective early diagnosis of heart disease, such as well changes of miRNAs content in blood serum are early even and happens before pathology progression. Experimental data from Lab bench as well as from patient's blood samples analysis would provide us a new knowledge which will be useful for completely new diagnosis system creation.

\section{Acknowledgements}

None.

\section{Conflict of interest}

The author declares no conflict of interest.

\section{References}

1. Townsend N, Wilson L, Bhatnagar P, et al. Cardiovascular disease in Europe: epidemiological update. Eur Heart J. 2016;37(42):3232-3245.

2. Hsieh PC, Segers VF, Davis ME, et al. Evidence from a genetic fate-mapping study that stem cells refresh adult mammalian cardiomyocytes after injury. Nat Med. 2007;13(8):970-974.

3. Roger VL, Go AS, Lloyd Jones DM, et al. Heart disease and stroke statistics--2012 update: a report from the American Heart Association. Circulation. 2012;125(1):e2-220.
4. Kontaridis MI, Geladari EV, Geladari CV. Pathways to myocardial hypertrophy. Int Cardiovasc Res J. 2014. p. 167-186.

5. Grigoryan T, Wend P, Klaus A, et al. Deciphering the function of canonical Wnt signals in development and disease: conditional lossand gain-of-function mutations of beta-catenin in mice. Genes Dev. 2008;22(17):2308-2341.

6. Dassanayaka S, Steven P. Jones recent developments in heart failure. Circ Res. 2015;117(7):e58-63.

7. Weinreuter M, Kreusser MM, Beckendorf J, et al. CaM kinase II mediates maladaptive post-infarct remodeling and pro-inflammatory chemo attractant signaling but not acute myocardial ischemia/reperfusion injury. EMBO Mol Med. 2014;6(10):1231-1245.

8. Lee R, Feinbaum R, Ambros V. A short history of a short RNA. Cell. 2004;116(2 Suppl):89-92.

9. Callis TE, Pandya K, Seok HY, et al. MicroRNA-208a is a regulator of cardiac hypertrophy and conduction in mice. J Clin Invest. 2009;119(9):2772-2786.

10. Luo X, Lin H, Pan Z, et al. Down-regulation of miR-1/miR-133 contributes to re-expression of pacemaker channel genes HCN2 and HCN4 in hypertrophic heart. J Biol Chem. 2008;283(29):20045-20052.

11. Shan H, Zhang Y, Lu Y, et al. Down regulation of miR-133 and miR-590 contributes to nicotine-induced atrial remodelling in canines. Cardiovasc Res. 2009;83(3):465-472.

12. Yang B, Lin H, Xiao J, et al. The muscle-specific microRNA miR-1 regulates cardiac arrhythmogenic potential by targeting GJA1 and KCNJ2. Nat Med. 2007;13(4):486-491.

13. Cheng Y, Tan N, Yang J, et al. A translational study of circulating cell-free microRNA-1 in acute myocardial infarction. Clin Sci. 2010;119(2):87-95.

14. Corsten MF, Dennert R, Jochems S, et al. Circulating microRNA-208b and microRNA-499 reflect myocardial damage in cardiovascular disease. Circ Cardiovasc Genet. 2010;3(6):499-506.

15. Fichtlscherer S, De Rosa S, Fox H, et al. Circulating microRNAs in patients with coronary artery disease. Circ Res. 2010;107(5):677-684.

16. Lutter D, Marr C, Krumsiek J, et al. Intronic microRNAs support their host genes by mediating synergistic and antagonistic regulatory effects. BMC Genomics. 2010;11:224.

17. vanRooij E, Olson EN. Searching for mir-acles in cardiac fibrosis. Circ Res. 2009;104(2):138-140.

18. Landskroner Eiger S, Qiu C, Perrotta P, et al. Endothelial miR-17 92 cluster negatively regulates arteriogenesis via miRNA-19 repression of WNT signaling. Proc Natl Acad Sci USA. 2015;112(41):12812-12817.

19. Mohamed JS, Lopez MA, Boriek AM. Mechanical stretch up-regulates microRNA-26a and induces human airway smooth muscle hypertrophy by suppressing glycogen synthase kinase-3b. J Biol Chem. 2010;285(38):29336-29347.

20. Adachi T, Nakanishi M, Otsuka Y, et al. Plasma microRNA 499 as a biomarker of acute myocardial infarction. Clin Chem. 2010;56(7):11831185 .

21. Gidlöf O, Andersson P, van der Pals J, et al. Cardio specific microRNA plasma levels correlate with troponin and cardiac function in patients with ST elevation myocardial infarction, are selectively dependent on renal elimination, and can be detected in urine samples. Cardiology. 2011;118(4):217-226.

22. Li H, Fan J, Yin Z, et al. Identification of cardiac-related circulating microRNA profile in human chronic heart failure. Oncotarget. 2015;7(1):33-45. 